\title{
Comparison: 3G Wireless Networks with 4G Wireless Networks Technology Wise
}

\author{
M. Ramzan ${ }^{1}$ and Javed Ahmed Shaheen ${ }^{2}$ \\ ${ }^{1}$ HOD CS Department \\ ${ }^{2}$ Ph.D (Scholar) GCUF \\ ${ }^{1,2}$ Computer Science Department, GC University Faisalabad \\ javedmatyana@gmail.com,ramzan.talib@gcuf.edu.pk
}

\begin{abstract}
Opportunities and challenges compel us to throw light on the differences between the two $3 G$ and $4 G$ networks. This task is being done in order to list out the drawbacks and merits of these $3 G$ and $4 G$ evolutions of the networks and further to implement $R \& D$ research to bring out their sparks in this modern era. If we look into $4 G$, objective of $4^{\text {th }}$ generation is to enhance specifications for a new radio-access technology for higher data rates, low latency and greater spectral efficiency so $4 G$ (also known as LTE-Advanced) is therefore not a new technology; it is actually an evolutionary step in the continuing development of LTE. The differences of $3 G$ and $4 G$ are done on the following Index terms: Background difference, definitions, technologies progress, speed/ rate of transmission, switching technologies used, network, band width, design specification, QoS, service and billing, features and capabilities.
\end{abstract}

\section{Keywords: SGSN, SONET, UTRAN, FLASH OFDM, RAN, CORE NETWORK}

\section{Introduction}

$3 \mathrm{G}$ is also called third generation. It is named as such because it is the third generation of the standards of telecommunication hardware. It is also the general technology for mobile networking, passing the recent $2.5 \mathrm{G}$. The technology is founded on the ITU or International Telecommunication Union group of standards which belongs to the IMT2000. 3G mobile telephony protocols support higher data rates, measured in kbps or Mbps intended for applications other than voice-centric and this shift from $2 \mathrm{G}$ to $3 \mathrm{G}$ not only gives users fresh ways to communicate but also a variety of new services such as browsing the Internet, e-mail, instant messaging, video-conferencing and digital television. 3G also makes guidance, presence, and location-based services available to mobile users as cameras and personal time management systems are expected to develop. $3 \mathrm{G}$ trials since 2001, across the world have shown that the main development from $2 \mathrm{G}$ and $2.5 \mathrm{G}$ to $3 \mathrm{G}$ is faster connection speed is referring to wider bandwidth. The uniqueness of $3 \mathrm{G}$ is to provide mobile multimedia services at a transmission rate of $144 \mathrm{kbps}$ at the high speed and $384 \mathrm{kbps}$ at the speed of walking and $2 \mathrm{Mbps}$ indoors. This theoretical maximum $2 \mathrm{Mbps}$ is close to the speed of LAN connections that many households nowadays have in their Networks. $4 \mathrm{G}$ is the fourth generation of mobile phone mobile communications standards. It is a successor of the third generation $(3 \mathrm{G})$ standards. A $4 \mathrm{G}$ system provides mobile ultra-broadband Internet access under LTE and WCDMA. The development of $4 \mathrm{G}$ is started in 2002 but the real new revolution started with 3GPP in December 1998. 3G systems are designed for multimedia communication that is for the higher data rates and new flexible communication capabilities of third generation systems

Received (July 15, 2017), Review Result (October 20, 2017), Accepted (October 21, 2017) 
[14].The next step was 3GPP Long-Term Evolution (LTE) with aims to improving the Universal Mobile Telecommunication System (UMTS), mobile phone standard to cope with future requirements. The LTE project is not a standard but it will result in the new evolved Release of the UMTS standard, including most or all of the extensions and modifications of the UMTS system. With the deployment of LTE wireless revolution will achieve a milestone i.e. for the first time; a wide-area wireless network will be universally deployed that has been primarily designed for IP based broadband data from the very beginning. LTE is rapidly becoming the dominant global standard for fourth generation cellular networks and all the major cellular players behind it and working toward its success. The 4G (fourth generation of cellular communication systems) is the emerging technology of future wireless networks.

\section{Background Difference}

In $3 \mathrm{~g}$ technology which is founded on the ITU or International Telecommunication Union group of a standard which belongs to the IMT-2000 use W-CDMA technology. It allows operators to provide users a bigger range of the latest services, as it gets bigger network capacity via heightened spectral efficiency. The included services are video calls, wide-area wireless voice telephone and broadband wireless information all included within the mobile environment. Whereas $4 \mathrm{G}$ technology which was started within cable television industry in 2009 which make users to explore new downloading speeds and capabilities. The utilization of LTE mobile broadband technology is an opportunity for the corporation to expand its horizons into $4 \mathrm{G}$ territory, upstaging current $3 \mathrm{G}$ capabilities. The necessity for $4 \mathrm{G}$ networks is associated with the increased utilization of data websites such as You Tube and Facebook, which require tremendous bandwidth in order to be used successfully.

\subsection{G Stands for 3rd Generation while 4G Stands for 4th Generation:}

$3 \mathrm{G}$ is currently the world's best connection method when it comes to mobile phones, and especially mobile Internet. $3 \mathrm{G}$ stands for 3rd generation as it is just that in terms of the evolutionary path of the mobile phone industry. 4G means 4th generation. This is a set of standard that is being developed as a future successor of $3 \mathrm{G}$ in the very near future.

\subsection{Architectural Difference:}

Both the Figures below provide the key components of these two architectures.

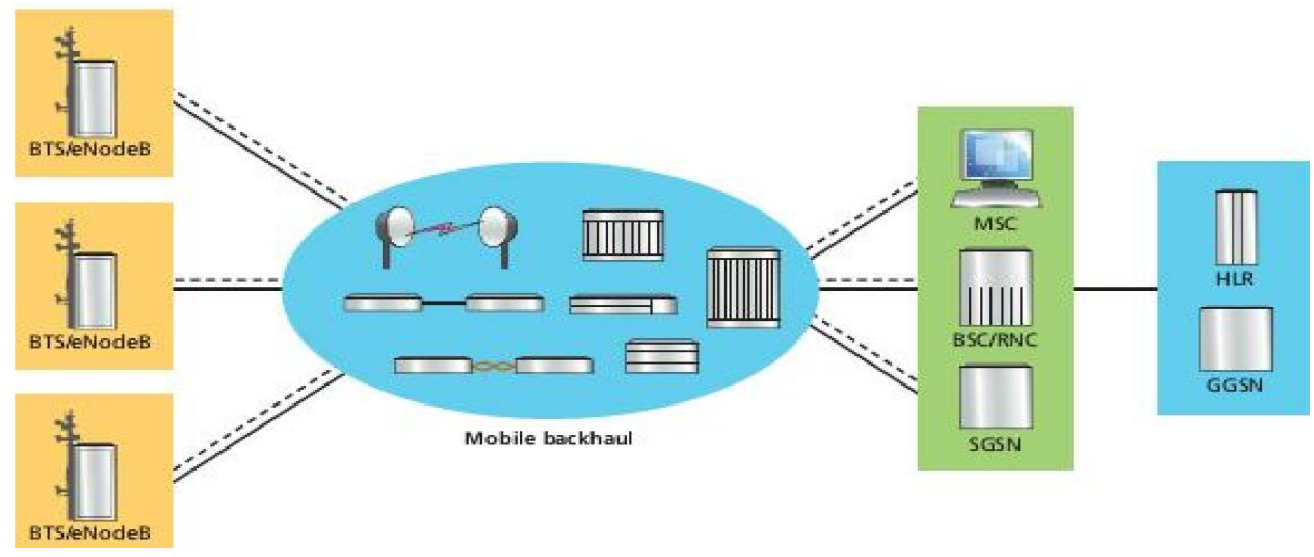

Figure 1. 3G Architecture 


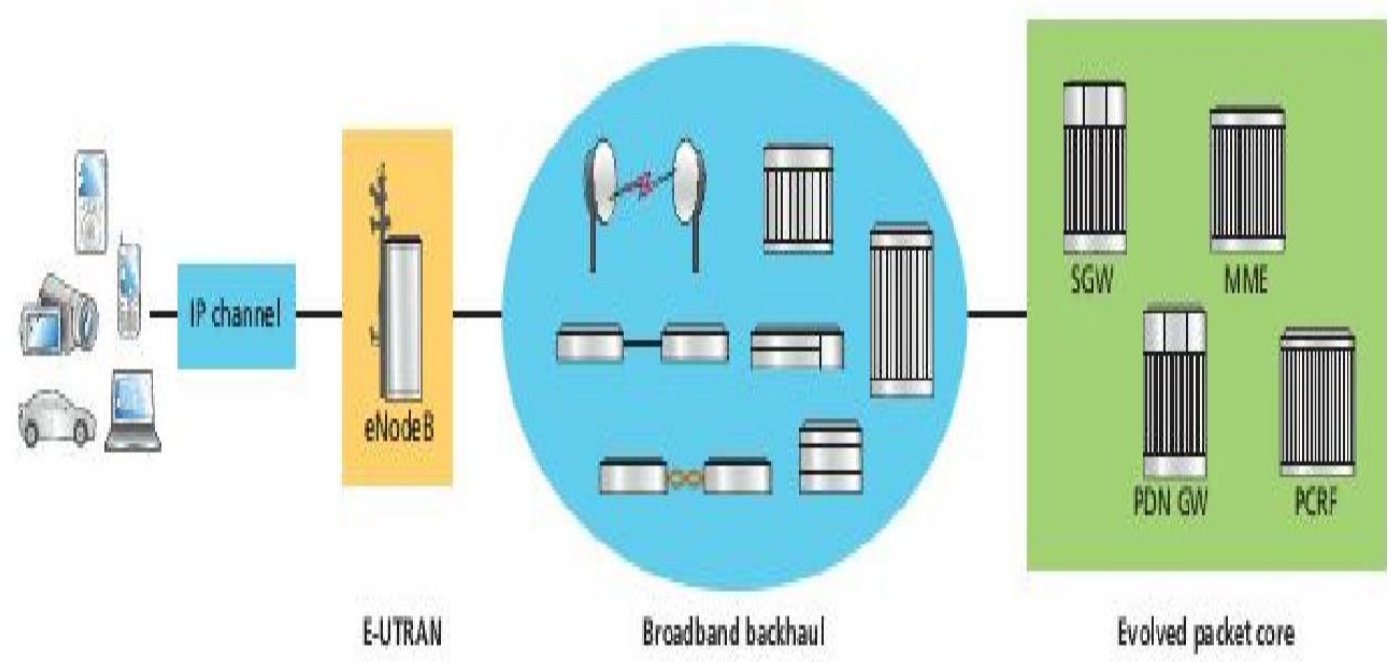

Figure 2. 4G Architecture

Several key differences in a LTE network enable more flexibility in its architecture than in a 3G. A functional representation of $3 \mathrm{G}$ network architecture is shown in Figure 1. In this network, the Base Terminal Station (BTS) or NodeBs aggregate the radio access network (RAN) traffic and transport it over a mobile backhaul network to the Radio Network Controllers (RNCs) or Base Station Controller (BSCs). Typically this transport is over T1/E1 copper facilities. If fiber is available at cell or near the cell site then the cell traffic is transported over SDH/SONET rings or more recently a carrier Ethernet network when the eNodeBs are equipped with IP/Ethernet interfaces. The bearer traffic from a number of RNCs/BSCs is multiplexed at the Mobile Telephone Switching Office (MTSO) and then transported via direct tunneling to the Gateway GPRS Serving Nodes (GGSNs) in the hub data center. This transport is normally over a SDH/SONET ring or a carrier Ethernet network. This tiered aggregation and transport structure lends itself to a point-topoint network topology to minimize both the amount of aggregation equipment required and the transport backhaul expense. In a $3 \mathrm{G}$ pre-Release 8 network, the RNCs and SGSNs are designed to support both the signaling and bearer plane processing and bandwidth requirements. The emphasis in the design for these network elements is in providing the processing necessary to support the high subscriber counts and Packet Data Protocol PDP contexts as the bandwidth requirements for delivery of the initial 3G data services (text and e-mail) were not significant. Since the data services that typically ran over these systems is not real-time neither QoS nor latency was an issue. Therefore, the placement of these elements is usually in locations that primarily meet the PDP context and network latency requirements. Thus, the current $3 \mathrm{G}$ packet core architecture is typically a centralized network design with the GGSNs deployed in major data centers, and all the data services are backhauled from the SGSNs which are strategically deployed in regional serving offices. Because the aggregate bandwidth for these services did not increase significantly until the past few years, the backhaul transport costs were manageable and could be supported with leased TDM or lower rate OC-n/STM-n interfaces.

Above Figure 2 provides a high-level functional representation of a LTE/4G network. This network is composed of three major sub-networks: the Evolved Universal Terrestrial Radio Access Networks (eUTRAN), which provides the air interface and local mobility management of the user equipment (UE), the evolved packet core (EPC), and the broadband backhaul network that provides the aggregation of cell traffic and transport back to the EPC. The 3GPP LTE standards defined the EPC as a set of logical data and control plane functions that can be implemented either as integrated or as separate network elements. The four EPC functions are: the Serving Gateway (SGW), the Packet 
Data Network Gateway (PGW) that supports the data or bearer traffic; and the Mobility Management Entity (MME) and the Policy Charging and Rules Function (PCRF) which support the dynamic mobility management and policy control traffic. The backhaul network either is owned by the wireless operator or is leased from a third party backhaul access provider. Any number of transport technologies can be used for backhaul including packet microwave, packet optical, Carrier Ethernet, IP/MPLS, GPON and xDSL.

\section{Network}

3G technologies are in widespread use while $4 \mathrm{G}$ compliant technologies are still in the horizon: The biggest difference between the two is in the existence of compliant technologies. There are a bunch of technologies that fall under 3G, including WCDMA, EV-DO, and HSPA among others. Although a lot of mobile phone companies are quick to dub their technologies as $4 \mathrm{G}$, such as LTE, WiMax, and UMB, none of these are actually compliant to the specifications set forth by the $4 \mathrm{G}$ standard. These technologies are often referred to as Pre-4G or 3.9G.

\subsection{Speed Comparison of $\mathbf{4 G}$ and $3 G$ :}

$4 \mathrm{G}$ speeds are meant to exceed that of $3 \mathrm{G}$. Current $3 \mathrm{G}$ speeds are topped out at $14 \mathrm{Mbps}$ downlink and 5.8Mbps uplink. To be able to qualify as a $4 \mathrm{G}$ technology, speeds of up to $100 \mathrm{Mbps}$ must be reached for a moving user and $1 \mathrm{Gbps}$ for a stationary user. So far, these speeds are only reachable with wired LANs. The fourth generation is faster, it is said to be four times faster than its predecessor. This allows for a connection speed more comparable to DSL and home cable networks. It is great news for those completing work and accomplishing important tasks away from their home and office. When uploading large documents and communicating via the internet, a fast connection is important whereas $3 \mathrm{G}$ doesn't favor such speed as compared to that of $4 \mathrm{G}$.

\begin{tabular}{|c|c|c|c|c|c|}
\hline Symbol & Standard & Full Name & $\begin{array}{l}\text { Maximum Download } \\
\text { Speed (Theoretical) }\end{array}$ & \multicolumn{2}{|c|}{$\begin{array}{l}\text { Maximum Upload } \\
\text { Speed (Theoretical) }\end{array}$} \\
\hline $2 \mathrm{G}$ & GSM & $\begin{array}{l}\text { Global System for Mobile } \\
\text { Communications }\end{array}$ & $14.4 \mathrm{Kbits} / \mathrm{s}$ & $14.4 \mathrm{Kbits} / \mathrm{s}$ & \\
\hline $\mathbf{G}$ & GPRS & $\begin{array}{l}\text { General Packet Radio } \\
\text { Service }\end{array}$ & $53.6 \mathrm{Kbits} / \mathrm{s}$ & $26.8 \mathrm{Kbits} / \mathrm{s}$ & \\
\hline $\mathbf{E}$ & EDGE & $\begin{array}{l}\text { Enhanced Data rates for } \\
\text { GSM Evolution }\end{array}$ & $217.6 \mathrm{Kbits} / \mathrm{s}$ & $108.8 \mathrm{Kbits} / \mathrm{s}$ & \\
\hline $3 G$ & UMTS & $\begin{array}{l}\text { Universal Mobile } \\
\text { Telecommunications System }\end{array}$ & $384 \mathrm{Kbits} / \mathrm{s}$ & $128 \mathrm{Kbits} / \mathrm{s}$ & \\
\hline $\mathbf{H}$ & HSPA & High-Speed Packet Access & $7.2 \mathrm{Mbits} / \mathrm{s}$ & 3.6 Mbits $/ \mathrm{s}$ & \\
\hline $\mathbf{H +}$ & HSPA+ & $\begin{array}{l}\text { Evolved High-Speed Packet } \\
\text { Access - Release } 6\end{array}$ & $14.4 \mathrm{Mbits} / \mathrm{s}$ & $5.76 \mathrm{Mbits} / \mathrm{s}$ & \\
\hline $\mathbf{H +}$ & HSPA+ & $\begin{array}{l}\text { Evolved High-Speed Packet } \\
\text { Access - Release } 7\end{array}$ & 21.1 Mbits/s or $28.0 \mathrm{Mbits} / \mathrm{s}$ & $11.5 \mathrm{Mbits} / \mathrm{s}$ & $\begin{array}{l}\text { Speed } \\
\text { Compar }\end{array}$ \\
\hline $\mathbf{H +}$ & HSPA+ & $\begin{array}{l}\text { Evolved High-Speed Packet } \\
\text { Access - Release } 8\end{array}$ & $42.2 \mathrm{Mbits} / \mathrm{s}$ & $11.5 \mathrm{Mbits} / \mathrm{s}$ & $\begin{array}{l}\text { ison } \\
\text { of All }\end{array}$ \\
\hline $\mathrm{H+}$ & HSPA+ & $\begin{array}{l}\text { Evolved High-Speed Packet } \\
\text { Access - Release } 9\end{array}$ & $84.4 \mathrm{Mbits} / \mathrm{s}$ & $11.5 \mathrm{Mbits} / \mathrm{s}$ & $\begin{array}{l}\text { Genera } \\
\text { tions }\end{array}$ \\
\hline $\mathbf{H +}$ & HSPA+ & $\begin{array}{l}\text { Evolved High-Speed Packet } \\
\text { Access - Release } 10\end{array}$ & $168.8 \mathrm{Mbits} / \mathrm{s}$ & $23.0 \mathrm{Mbits} / \mathrm{s}$ & and $4 \mathrm{G}$ \\
\hline $4 G$ & LTE & Long Term Evolution & $100 \mathrm{Mbits} / \mathrm{s}$ & $50 \mathrm{Mbits} / \mathrm{s}$ & \\
\hline $4 G$ & LTE-A & $\begin{array}{l}\text { Long Term Evolution - } \\
\text { Advanced }\end{array}$ & $1 \mathrm{Gbits} / \mathrm{s}$ & $500 \mathrm{Mbits} / \mathrm{s}$ & \\
\hline
\end{tabular}

Figure 3. 3G and 4G Speed Comparison 


\subsection{Switching Techniques in $3 G$ and $4 G$}

Another key change in $4 \mathrm{G}$ is the abandonment of circuit switching. $3 \mathrm{G}$ technologies use a hybrid of circuit switching and packet switching. Circuit switching is a very old technology that has been used in telephone systems for a very long time. The downside to this technology is that it ties up the resource for as long as the connection is kept up. Packet switching is a technology that is very prevalent in computer networks but has since appeared in mobile phones as well. With packet switching, resources are only used when there is information to be sent across. The efficiency of packet switching allows the mobile phone company to squeeze more conversations into the same bandwidth. $4 \mathrm{G}$ technologies would no longer utilize circuit switching even for voice calls and video calls. All information that is passed around would be packet switched to enhance efficiency.

\subsection{Internetworking of 3G and 4G:}

Another difference between the two is the network. When the $3 \mathrm{G}$ was introduced, cell phone users were finally able to talk and access data at the same time and with higher data rates. This allowed for a better full service for cell phone users wishing to access the internet. And what is even greater is the $4 \mathrm{G}$ data rates are expected to be even higher. Users will have the capability of accessing more data at higher speeds while talking on their cell phone. In addition, the fourth generation permits more data transmission of such services as games and multimedia. It also allows a larger amount of internet support.

\subsection{Bandwidth Difference:}

The next difference between the third and fourth generation to is bandwidth. At first glance, the bandwidth of both $3 \mathrm{G}$ and $4 \mathrm{G}$ are the same, numbered at between 5 and 20 MHz. However, the rate of data is what makes the difference between the two. While the data rate of the third generation only goes up to $2 \mathrm{Mbps}$, the fourth goes all the way up to between $100 \mathrm{Mbps}$ to $1 \mathrm{Gbps}$.

\section{Design specification:}

The 3G technology provides both circuit design and packet design. Circuit design, being the oldest, has greater ability to hold the connection for a longer duration. On the other hand the packet design is a wireless technology and is the core part of internet data transmission. The combination of these two patterns helps 3G technology to perform better and faster. However, the $4 \mathrm{G}$ technology is kept free from circuit design with an intention to gives nanosecond wings to data transfer and so has packet design only.

\subsection{Data transmission rate}

$3 \mathrm{G}$ system is based on wideband CDMA that operates in $5 \mathrm{MHz}$ of bandwidth and can produce download data rates of typically $384 \mathrm{~kb} / \mathrm{s}$ under normal conditions and up to 2 $\mathrm{Mb} / \mathrm{s}$ in some instances. $3 \mathrm{~g}$ phone standards have been expanded and enhanced to further expand data speed and capacity. The WCDMA phones have added high speed packet access (HSPA) that use higher level QAM modulation to get speeds up to 21 or $42 \mathrm{Mb} / \mathrm{s}$ downlink (cell site to phone) and up to 7 and/or $14 \mathrm{Mb} / \mathrm{s}$ uplink (phone to cell site).whereas in $4 \mathrm{G}$ also known as LTE uses a completely different radio technology. Instead of CDMA, it uses orthogonal frequency division multiplexing (OFDM) and OFDM access. This modulation technique divides a channel usually 5,10 or $20 \mathrm{MHz}$ wide into smaller subchannels or subcarriers each $15 \mathrm{kHz}$ wide. Each is modulated with part of the data. The fast data is divided into slower streams that modulate the subcarriers with one of several modulation schemes like QPSK or 16QAM. It also defines multiple input multiple output (MIMO) operation that uses several transmitter- 
receiver-antennas. The data stream is divided between the antennas to boost speed and to make the link more reliable. Using OFDM and MIMO lets LTE deliver data at a rate to $100 \mathrm{Mb} / \mathrm{s}$ downstream and $50 \mathrm{Mb} / \mathrm{s}$ upstream under the best conditions. In $4 \mathrm{G}$ the theoretical upper data rate is $1 \mathrm{~GB} / \mathrm{s}$. That remains to be seen in practice.

\subsection{Quality of service:}

In 3G, network based Qos depends on following factor to provide a satisfactorily service as: Throughput, Packet Loss Rate, Packet Loss Rate, reliability and delay. Where as in $\mathbf{4 G}$ With respect to network quality, many telecommunications providers are promising that there will be enhanced connectivity, and the quality of data that is transmitted across the network will be of the highest possible quality. The main challenge that $4 \mathrm{G}$ networks are facing is integrating non-IP-based and IP-based devices. It is known that devices that are not IP address based are generally used for services such as VoIP. On the other hand, devices that are IP address based are used for data delivery. 4G networks will serve both types of devices.

\subsection{Service and Billing:}

$3 \mathrm{G}$ networks that are capable of supporting an ever-increasing variety of data services from streaming video, to gaming, to proprietary business applications, to mobile commerce transactions for tangible goods and services. However, as 3G finally makes it into the mainstream, its success is inextricably linked to how the CSPs (Communications Service Providers) charge and bill for services in ways that are both intuitive and acceptable to the end user while also being relevant to the CSP's costs and billing capabilities Where as in $4 \mathrm{G}$ managing user accounts and billing them has become much more complicated with $4 \mathrm{G}$ networks. This is mainly due to heterogeneity of $4 \mathrm{G}$ networks and the frequent interaction of service providers.

\subsection{Features and capabilities:}

$3 \mathrm{G}$ has features with Speed of mobile communication in 3G ranges from 600-800 $\mathrm{Kbit} / \mathrm{sec}$. Also it provides high quality wireless sound and facilitates with global roaming. It accommodates distance surveillance and enables mobile TV Whereas the ambitious goal of $4 \mathrm{G}$ is to allow everyone to access the Internet anytime and everywhere. The provided connection to Internet will allow users to access all type of services including text, databases, and multimedia. 4G will also provide higher bandwidth, data rate, lower authentication overhead, and will ensure the service is constantly provided to the user without any disruption.

Table 1. Comparison of Different Aspects of 3G and 4G

\begin{tabular}{|l|l|l|}
\hline Specifications & $3 \mathrm{G}$ & $4 \mathrm{G}$ \\
\hline Frequency Band & $1.8-2.5 \mathrm{GHz}$ & $2-8 \mathrm{GHz}$ \\
\hline Bandwidth & $5-20 \mathrm{MHz}$ & $5-20 \mathrm{MHz}$ \\
\hline Data rate & Up to $2 \mathrm{Mbps}$ & $20 \mathrm{Mbps}$ or more \\
\hline Access & Wideband CDMA & Multi-carrier - CDMA or OFDM(TDMA) \\
\hline FEC & Turbo-codes & Concatenated codes \\
\hline Switching & Circuit/Packet & Packet \\
\hline
\end{tabular}


Table2: Comparison in brief

\begin{tabular}{|l|l|l|}
\hline Attributes $\downarrow$ & $3 \mathrm{G}$ & $4 \mathrm{G}$ \\
\hline Data Throughput: & Up to 3.1mbps & $\begin{array}{l}\text { 3to5mbps but potential estimated at } \\
\text { a range of } 10 \text { to } 300 \text { mbps. }\end{array}$ \\
\hline Peak Upload Rate: & $50 \mathrm{Mbit} / \mathrm{s}$ & $50 \mathrm{Mbit} / \mathrm{s}$ \\
\hline $\begin{array}{l}\text { Peak Download } \\
\text { Rate: }\end{array}$ & $100 \mathrm{Mbit} / \mathrm{s}$ & $1 \mathrm{Gbit} / \mathrm{s}$ \\
\hline $\begin{array}{l}\text { Switching } \\
\text { Technique: }\end{array}$ & $\begin{array}{l}\text { packet switching } \\
\text { /circuit switch }\end{array}$ & $\begin{array}{l}\text { packet switching, message } \\
\text { switching }\end{array}$ \\
\hline $\begin{array}{l}\text { Network } \\
\text { Architecture: }\end{array}$ & Wide Area Cell Based & $\begin{array}{l}\text { Integration of wireless LAN and } \\
\text { Wide area. }\end{array}$ \\
\hline $\begin{array}{l}\text { Services And } \\
\text { Applications: }\end{array}$ & $\begin{array}{l}\text { CDMA 2000, UMTS, } \\
\text { EDGE etc }\end{array}$ & Wimax2 and LTE-Advance \\
\hline $\begin{array}{l}\text { Forward error } \\
\text { correction (FEC): }\end{array}$ & $\begin{array}{l}\text { 3G uses Turbo codes } \\
\text { for error correction }\end{array}$ & $\begin{array}{l}\text { Concatenated codes are used for } \\
\text { error correctionsin4G. }\end{array}$ \\
\hline Frequency Band: & $1.8-2.5 \mathrm{GHz}$ & $2-8 \mathrm{GHz}$ \\
\hline
\end{tabular}

\section{Applications of 3G and 4G}

The emerging applications for $3 \mathrm{G}$ and $4 \mathrm{G}$ wireless systems typically require highly Heterogeneous and time varying quality of service from the underlying protocol layers. So adaptability will be one of the basic requirements to the development and delivery of new mobile services. Promising techniques and possible topics may include: Mobile application should refer to a user's profile so that it can be delivered in a way most preferred by the subscriber, such as context-based personalized services. This also brings the applications with adaptability to terminals that are moving in varying locations and speeds. Techniques such as adaptive multimedia and unified messaging take the terminal characteristics into account and ensure that the service can be received and run on a terminal with the most suitable form to the host type. The $4 \mathrm{G}$ technology will be able to support Interactive services like Video Conferencing (with more than 2 sites simultaneously), Wireless Internet, etc. The bandwidth would be much wider (100 MHz) and data would be transferred at much higher rates. The cost of the data transfer would be comparatively very less and global mobility would be possible. The networks will be all IP networks based on IPv6. The antennas will be much smarter and improved access technologies like OFDM and MC-CDMA (Multi Carrier CDMA) will be used. Also the security features will be much better. Long-Term (Radio) Evolution or LTE is also part of $3 \mathrm{G}$ technology. It's a $3 \mathrm{GPP}$ its research item for Release 8. It's also known as $3.9 \mathrm{G}$ or "Super 3G" by some researchers. It's planned to commercialize in 2009. It was aims at peak data rates of $200 \mathrm{Mbps}$ (DL) and $100 \mathrm{Mbps}$ (UL).

\section{Multiple Access Techniques in 3G \& 4G}

3G wireless multiple access techniques are widely based on CDMA and WCDMA. But $4 \mathrm{G}$ demands a better multiple access technique for reducing the MAI (Multiple Access Interference) and ISI (Inter Symbol Interference) and thus improve the bit error rate performance. MC-CDMA is the best candidate that would satisfy the demands of $4 \mathrm{G}$ 
wireless systems. Moreover adaptive modulation techniques have been proposed for $4 \mathrm{G}$, where the modulation scheme is changed dynamically based on the current channel estimates. MCCDMA is the hybrid combination of OFDM (Orthogonal Frequency Division Multiplexing) and CDMA. MC-CDMA with adaptive modulation promises to meet the demands of $4 \mathrm{G}$ regarding high data rate with a lower BER (Bit Error Rate). OFDM has the capability to cancel multipath distortion in a spectrally efficient manner. Rapid variation in channel characteristics are caused by multi-path and Doppler spread (due to the different speeds of mobile). Sometimes these time varying channels are characterized by very good SNR (Signal to Noise Ratio), but worse SNR at other times. So a fixed modulation technique cannot achieve the best Spectral efficiency as the system has to be built with a modulation scheme considering the worst case scenario. Hence during good channel conditions the system would not be able to obtain the best possible spectral efficiency. This is where adaptive modulation shows its role. Adaptive Modulation techniques takes advantage of the time varying channel characteristics and adjust the transmission power, data rate, coding and modulation scheme for the best spectral efficiency.

\section{Multimedia \& Video Services in 3G/4G}

Wireless systems are expected to deliver efficient multimedia services at very high data rates. Basically there are two types of video services: bursting and streaming video Services. Streaming is performed when a user requires real time video services, in which the server delivers data continuously at a playback rate. Streaming has little memory requirement as compared to bursting. The drawback of streaming video is that it does not take advantage of available Bandwidth. Even if the entire system bandwidth is available for the user, streaming video service will transmit data only at a particular playback rate. Bursting is basically file downloading using a buffer and this is done at the highest data rate taking advantage of the whole available bandwidth. The flaw with this type of transmission is that it demands a large memory requirement. So work is being done to come up with a new scheme that limits the memory requirements and can exploit the available bandwidth of the system.

\section{Applications of $4 \mathrm{G}$ which are not present in $3 G$}

\subsection{Virtual Presence:}

This means that $4 \mathrm{G}$ provides user services at all times, even if the user is off-site. Virtual navigation is also embedded in $4 \mathrm{G}$ mean provides users with virtual navigation through which a user can access a database of the streets, buildings etc of large cities. This requires high speed data transmission.

\subsection{Tele-Medicine:}

$4 \mathrm{G}$ will support remote health monitoring of patients. A user need not go to the hospital instead a user can get videoconference assistance for a doctor at anytime and anywhere.

\subsection{Tele-Geoprocessing Applications:}

This is a combination of GIS (Geographical Information System) and GPS (Global Positioning System) in which a user can get the location by querying. 


\section{Conclusion}

For better performance we have to make $3 \mathrm{G}$ as IP based which will allow higher data transmission rate. We have to use only packet switching so that we can achieve higher internet speed eliminating circuit switching which makes internet speed slow. To have the better performance we have to use OFDM technology with QPSK and 16 QAM modulation rather than simple QAM modulation technology. The $3 \mathrm{G}$ should be integrated with the IP based technology so that it can have the tremendous data transmission and support VoIP as well. We have to increase the band width of the $3 \mathrm{G}$ networks by using technology such as orthogonal frequency division if we are able to integrate it with the wireless LAN for better quality of service. At an early stage the Flash-OFDM (Fast lowlatency access with seamless handoff orthogonal frequency division multiplexing (FlashOFDM also referred to as F-OFDM) system was expected to be further developed into a 4G standard. No source suggests peak download and upload rates of more than the $1 \mathrm{Gbps}$ to be offered by ITU-R's definition of $4 \mathrm{G}$ systems. If any further generation appears, and reflects these prognoses, the major difference from a user point of view between $4 \mathrm{G}$ and next Generation techniques must be something else than increased maximum throughput; for example lower battery consumption, lower outage probability / better coverage, high bit rates in larger portions of the coverage area, cheaper or no traffic fees due to low infrastructure deployment costs, or higher aggregate capacity for many simultaneous users i.e., higher system level spectral efficiency.

\section{References}

[1] TSG RAN wg4 tr25.942 version 1.0.0, wireless intelligent network, (2004) March.

[2] J. Thorner, "Intelligent network", Artech House, (1994).

[3] H. Gobjuka, "4G wireless Networks Opportunities and challenges", Verizon ,919 hidden Ridge Irving, TX75038.

[4] R. Prasad and L. Munoz, "WLAN and WPANs towards 4G Wireless", Artech House, (2003) March.

[5] J. Thorer, "Intelligent network", (1994), Artech House.

[6] S. Tabbane, "An alternative strategy for location tracking", IEEE, J select Areas commun, vol. 13, no. 5, (1995) June, pp. 880-892.

[7] TRA, "3G Network Aspects", Understanding 3G Wireless Mobile Communications Proceedings, (1998).

[8] S. Bali and J. Korah, "Quality of Service in 3G Wireless Networks", a project paper submitted to ECE5556, Virginia Tech, (2001).

[9] R. Prasad and T. Ojanpera, "An Overview of CDMA Evolution toward Wideband CDMA", IEEE Communications Surveys, Fourth Quarter, vol. 1, no. 1, (1988).

[10] "first in the world with 4G services", TeliaSonera. 14 December 2009. Retrieved 2010-09-06.

[11] D. Morr, (June 9, 2009), "Verizon mandates IPv6 support for next-gen cell phones", Retrieved June 10, 2009.

[12] P. Zheng, L. Peterson, B. Davie and A. Farrel, "Wireless Networking Complete", Morgan Kaufmann, (2009).

[13] J. Alabaster, "Japan's NTT DoCoMo signs up 1 million LTE users in a month, hits 5 million total", Network World. IDG. Archived from the original on December 3, 2013, (2012) August 20.

[14] H. Holma and A. Toskala, "WCDMA for UMTS", Wiley Online Library, vol. 4.

\section{Authors}

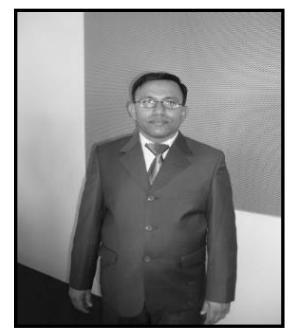

Dr. M. Ramzan Talib, Chairperson, Computer Science Department GCU Faisalabad 
International Journal of Advanced Science and Technology

Vol.108 (2017)

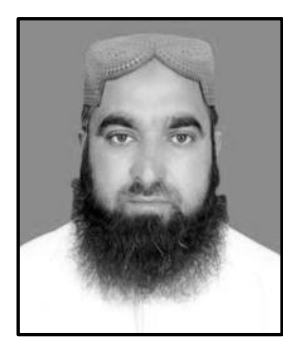

Javed Ahmed Shaheen is presently Studying Ph.D (CS) in from GC University Faisalabad Pakistan under the supervision of sir M.Ramzan sb. 